\title{
Nonsequential double ionization with time-dependent renormalized-natural-orbital theory
}

\author{
M. Brics, J. Rapp, and D. Bauer \\ Institut für Physik, Universität Rostock, 18051 Rostock, Germany
}

(Dated: September 19, 2021)

\begin{abstract}
Recently introduced time-dependent renormalized-natural-orbital theory (TDRNOT) is tested on nonsequential double ionization (NSDI) of a numerically exactly solvable one-dimensional model He atom subject to few-cycle, 800-nm laser pulses. NSDI of atoms in strong laser fields is a prime example of non-perturbative, highly correlated electron dynamics. As such, NSDI is an important "worst-case" benchmark for any timedependent few and many-body technique beyond linear response. It is found that TDRNOT reproduces the celebrated NSDI "knee," i.e., a many-order-of-magnitude enhancement of the double ionization yield (as compared to purely sequential ionization) with only the ten most significant natural orbitals (NOs) per spin. Correlated photoelectron spectra—as "more differential" observables—require more NOs.

PACS numbers: $31.15 . e e, 32.80 . \mathrm{Rm}, 31.70 . \mathrm{Hq}$
\end{abstract}

\section{INTRODUCTION}

Nonsequential double ionization (NSDI) in intense laser pulses has been experimentally observed in measurements of ion yields as a function of the laser intensity, which deviate from the yields expected from a sequential ionization scenario, forming the so-called NSDI "knee" (see $[1,2]$ for recent reviews). In fact, the multiple ionization yields are typically enhanced by several orders of magnitude. With ionization yields being rather integrated observables the mechanism behind NSDI could not be unequivocally resolved until the measurement of ion spectra $[3,4]$ and correlated photoelectron distributions has become feasible (see $[5,6]$ for early and, e.g., [7] for very recent work). Meanwhile NSDI is understood in terms of a recollision process: one electron is emitted but oscillates back to its parent ion to knock out the next electron. If the return energy is not sufficient for collisional ionization, the next electron might be excited and later emitted owing to the laser field [8].

The described recollision scenario poses a huge challenge for general many-body methods when applied to such a fewelectron test case. For example, in time-dependent HartreeFock (TDHF) or time-dependent density functional theory (TDDFT) applied to He starting from the singlet ground state there is only one spatial orbital describing both electrons (one spin-up, the other spin-down). Not surprisingly, it was found that such methods are not capable of describing NSDI [9], although formally for different reasons. TDHF, as a meanfield approach, does not incorporate correlation by definition. TDDFT is in principle exact but only in the sense that it gives the exact time-dependent electron density. However, even if the exact time-dependent electron density was known from a TDDFT calculation employing the exact exchange-correlation potential [10], the exact double-ionization probability could still not be calculated because this observable is unknown as an explicit functional of the electron density, and simple approximations to it do not reproduce the NSDI knee [11, 12].

Solving the full time-dependent Schrödinger equation (TDSE) for He in full dimensionality and in strong, longwavelength (i.e., $\geq 800 \mathrm{~nm}$ ) laser fields is still beyond what is possible with current super computer technology [13]. Therefore it is essential to develop practicable time-dependent many-electron methods beyond linear response that account for correlation. Time-dependent configuration interaction (TDCI) [14, 15], multiconfigurational time-dependent Hartree (MCTDH) [16] or multiconfigurational TDHF (MCTDHF) [17-20] are exact in principle. However, the crucial question in practice is how many configurations or determinants are required to recover a certain strong correlation feature such as the NSDI knee. General conclusions are difficult to draw, as different TDCI and MCTDHF approaches may vary in the single-particle basis functions chosen. It has been shown that for $\mathrm{He}\left(\right.$ or $\mathrm{H}_{2}$ ) a time-dependent variational approach using a wavefunction ansatz with two different single-particle orbitals (time-dependent extended Hartree-Fock) [9, 21] or an ad hoc ansatz with an "inner" and an "outer" outer orbital [22] at least generate kneelike structures in the double-ionization yield. However, they are only in poor agreement with the exact numerical results available for low-dimensional models. To the best of our knowledge there are no systematic tests of computational approaches that demonstrate a convergence toward the exact NSDI knee. In fact, we are not aware of any work that accurately reproduces the NSDI knee using a manybody method that overcomes the "exponential wall" [23]. In this work, we will provide such an analysis for our recently introduced time-dependent renormalized-natural-orbital theory (TDRNOT) [24, 25].

\section{THEORY}

Before we present results on the NSDI knee (Sec. III A) and on correlated photoelectron spectra (Sec. III B), we briefly introduce the He model, review the essentials of TDRNOT, particularly when applied to a two-electron system, and discuss the observables to be calculated.

Atomic units (a.u.) are used unless otherwise indicated. 


\section{A. Model atom}

The widely applied one-dimensional helium model atom [9, $10,12,20,26-29]$ in a laser field has the Hamiltonian

$$
\hat{H}^{(1,2)}(t)=\hat{h}^{(1)}(t)+\hat{h}^{(2)}(t)+v_{\mathrm{ee}}^{(1,2)}
$$

where upper indices indicate the action on either electron 1 , electron 2, or both. The single-particle Hamiltonian reads $\hat{h}(t)=\hat{H}_{A}+\hat{H}_{L}(t)$, with

$$
\hat{H}_{A}=\frac{\hat{p}^{2}}{2}-\frac{2}{\sqrt{x^{2}+\varepsilon_{\mathrm{ne}}}},
$$

$\hat{H}_{L}(t)=A(t) \hat{p}$ (dipole approximation and velocity gauge with the $A^{2}$ term transformed away), and the electron-electron interaction

$$
v_{\mathrm{ee}}^{(1,2)}=\frac{1}{\sqrt{\left(x^{(1)}-x^{(2)}\right)^{2}+\varepsilon_{\mathrm{ee}}}} .
$$

The electron-ion smoothing parameter $\varepsilon_{\text {ne }}=0.50$ is chosen such that the groundstate energy of $\mathrm{He}^{+} E_{0}^{\mathrm{He}^{+}}=-2.0$ is recovered. The electron-electron smoothing parameter $\varepsilon_{\mathrm{ee}}=$ 0.33 is tuned to yield the neutral-He energy $E_{0}^{\mathrm{He}}=-2.9$.

\section{B. Density matrices, renormalized-natural-orbitals, and their equations of motion}

The Hamiltonian (1) does not act on the spin, which-in the two-particle case-allows one to factorize the wavefunction,

$$
\langle 12 \mid \Phi(t)\rangle=\Phi(12 ; t)=\Phi\left(x_{1} x_{2} ; t\right) \Phi_{\sigma_{1} \sigma_{2}} .
$$

Here 1 and 2 are shorthand notations for position and spin $\left(x_{1}, \sigma_{1}\right)$ and $\left(x_{2}, \sigma_{2}\right)$, respectively. The two- and one-body density matrices read

$$
\begin{gathered}
\gamma_{2}\left(12,1^{\prime} 2^{\prime} ; t\right)=\Phi(12 ; t) \Phi^{*}\left(1^{\prime} 2^{\prime} ; t\right) \\
\gamma_{1}\left(1,1^{\prime} ; t\right)=2 \int \mathrm{d} 2 \gamma_{2}\left(12,1^{\prime} 2 ; t\right) .
\end{gathered}
$$

Natural orbitals (NOs) $\phi_{k}(1 ; t)=\langle 1 \mid k(t)\rangle$ are defined as eigenvectors of $\gamma_{1}$ :

$$
\gamma_{1}\left(1,1^{\prime} ; t\right)=\sum_{k} n_{k}(t) \phi_{k}(1 ; t) \phi_{k}^{*}\left(1^{\prime} ; t\right) .
$$

The corresponding eigenvalues $n_{k}(t) \in[0,1]$ are called occupation numbers (ONs). NOs and ONs were introduced a long time ago (see, e.g., [30-32]), but only recently have their usefulness for time-dependent few- and many-body problems been studied [33-38].

The coupled equations of motion for the ONs and the NOs can be unified by introducing renormalized NOs (RNOs) [24]

$$
\langle 1 \mid \tilde{k}(t)\rangle=\tilde{\phi}_{k}(1 ; t)=\sqrt{n_{k}(t)} \phi_{k}(1 ; t)
$$

so that

$$
n_{k}(t)=\langle\tilde{k}(t) \mid \tilde{k}(t)\rangle
$$

and

$$
\gamma_{1}\left(1,1^{\prime} ; t\right)=\sum_{k} \tilde{\phi}_{k}(1 ; t) \tilde{\phi}_{k}^{*}\left(1^{\prime} ; t\right) .
$$

The two-body density matrix expanded in RNOs reads

$$
\begin{aligned}
& \gamma_{2}\left(12,1^{\prime} 2^{\prime} ; t\right) \\
= & \sum_{i j k l} \tilde{\gamma}_{2, i j k l}(t) \tilde{\phi}_{i}(1 ; t) \tilde{\phi}_{j}(2 ; t) \tilde{\phi}_{k}^{*}\left(1^{\prime} ; t\right) \tilde{\phi}_{l}^{*}\left(2^{\prime} ; t\right) .
\end{aligned}
$$

The equation of motion (EOM) for the RNOs is [25]

$$
\begin{aligned}
\mathrm{i} \partial_{t}|\tilde{n}\rangle=\hat{h}(t)|\tilde{n}\rangle+\mathcal{A}_{n}(t)|\tilde{n}\rangle & \\
& +\sum_{k \neq n} \mathcal{B}_{n k}(t)|\tilde{k}\rangle+\sum_{k} \hat{\mathcal{C}}_{n k}(t)|\tilde{k}\rangle
\end{aligned}
$$

with

$$
\begin{aligned}
\mathcal{A}_{n}(t) & =-\frac{1}{n_{n}(t)} \operatorname{Re} \sum_{j k l} \tilde{\gamma}_{2, n j k l}(t)\left\langle\tilde{k} \tilde{l}\left|v_{\mathrm{ee}}\right| \tilde{n} \tilde{j}\right\rangle, \\
\mathcal{B}_{n k}(t) & =\frac{2}{n_{k}(t)-n_{n}(t)} \sum_{j p l}\left[\tilde{\gamma}_{2, k j p l}(t)\left\langle\tilde{p} \tilde{l}\left|v_{\mathrm{ee}}\right| \tilde{n} \tilde{j}\right\rangle\right. \\
& \left.-\tilde{\gamma}_{2, p \ln j}(t)\left\langle\tilde{k} \tilde{j}\left|v_{\mathrm{ee}}\right| \tilde{p} \tilde{l}\right\rangle\right],
\end{aligned}
$$

and

$$
\hat{\mathcal{C}}_{n k}(t)=2 \sum_{j l} \tilde{\gamma}_{2, k j n l}(t)\left\langle\tilde{l}\left|v_{\mathrm{ee}}\right| \tilde{j}\right\rangle
$$

One observes that the effective Hamiltonian in the TDSE-like equation (12) consists of the usual one-body operator $\hat{h}(t)$, a diagonal part $\mathcal{A}_{n}(t) \in \mathbb{R}$, the part $\mathcal{B}_{n k}(t) \in \mathbb{C}$ which couples RNOs, and the operator $\hat{\mathcal{C}}_{n k}(t)$, which also couples RNOs. As the effective Hamiltonian in (12) is Hermitian, the corresponding time evolution of the RNOs is unitary.

In general, there are infinitely many NOs required to describe a correlated quantum system, even if it contains only two particles. Ordered decreasingly according to their ONs, the number of RNOs taken into account in an actual numerical implementation of (12) is necessarily truncated, which introduces errors in the propagation. The effect of this truncation will be seen in the results in Sec. III below.

In the two-particle case the expansion coefficients $\tilde{\gamma}_{2, i j k l}(t)$ are exactly known [25],

$$
\tilde{\gamma}_{2, i j k l}(t)=(-1)^{i+k} \frac{e^{\mathrm{i}\left[\varphi_{i}-\varphi_{k}\right]}}{2 \sqrt{n_{i}(t) n_{k}(t)}} \delta_{i, j^{\prime}} \delta_{k, l^{\prime}}
$$

Here, the "prime operator" acts on the positive integer $k$ according to

$$
k^{\prime}= \begin{cases}k+1 & \text { if } k \text { odd } \\ k-1 & \text { if } k \text { even }\end{cases}
$$


and the phase factors are [25]

$$
e^{\mathrm{i} \varphi_{i}^{(\mathrm{S})}}=2 \delta_{k, 1}+2 \delta_{k, 2}-1, \quad e^{\mathrm{i} \varphi_{i}^{(\mathrm{T})}}=1
$$

in the spin-singlet and -triplet case, respectively. Note that the EOM for the RNOs (12) is given here for phase-including NOs [35] so that $\varphi_{i}$ and $\varphi_{k}$ in (16) do not depend on time. Employing the factorization (4) we can write

$$
\begin{gathered}
\gamma_{2}\left(12,1^{\prime} 2^{\prime} ; t\right)=\Phi\left(x_{1} x_{2} ; t\right) \Phi^{*}\left(x_{1}^{\prime} x_{2}^{\prime} ; t\right) \Phi_{\sigma_{1} \sigma_{2}} \Phi_{\sigma_{1}^{\prime} \sigma_{2}^{\prime}}^{*}, \\
\gamma_{1}\left(1,1^{\prime} ; t\right)=\gamma_{1}\left(x_{1}, x_{1}^{\prime} ; t\right) \sum_{\sigma_{2}} \Phi_{\sigma_{1} \sigma_{2}} \Phi_{\sigma_{1}^{\prime} \sigma_{2}}^{*}
\end{gathered}
$$

where

$$
\begin{aligned}
\gamma_{1}\left(x_{1}, x_{1}^{\prime} ; t\right) & =2 \int \mathrm{d} x_{2} \gamma_{2}\left(x_{1} x_{2}, x_{1}^{\prime} x_{2} ; t\right) \\
& =\sum_{k} \underline{n}_{k}(t) \underline{\phi}_{k}\left(x_{1} ; t\right) \underline{\phi}_{k}^{*}\left(x_{1}^{\prime} ; t\right) \\
& =\sum_{k} \underline{\tilde{\phi}}_{k}\left(x_{1} ; t\right) \underline{\tilde{\phi}}_{k}^{*}\left(x_{1}^{\prime} ; t\right), \\
\gamma_{2}\left(x_{1} x_{2}, x_{1}^{\prime} x_{2}^{\prime} ; t\right) & =\Phi\left(x_{1} x_{2} ; t\right) \Phi^{*}\left(x_{1}^{\prime} x_{2}^{\prime} ; t\right) \\
& =\sum_{i j k l} \tilde{\underline{q}}_{2, i j k l}(t) \underline{\tilde{\phi}}_{i}\left(x_{1} ; t\right) \underline{\tilde{\phi}}_{j}\left(x_{2} ; t\right) \\
& \quad \times \underline{\tilde{\phi}}_{k}^{*}\left(x_{1}^{\prime} ; t\right) \underline{\tilde{\phi}}_{l}^{*}\left(x_{2}^{\prime} ; t\right) .
\end{aligned}
$$

Here and in the following, spatial RNOs and quantities calculated from them (e.g., $\left.\underline{n}_{i}(t)=\left\langle\tilde{\phi}_{i}(t) \mid \tilde{\phi}_{i}(t)\right\rangle\right)$ will be indicated by underlining them. How the RNOs can be written as a factorization in the spatial and the spin part is discussed in detail in [25]. In this work we will only consider results for the singlet configuration where the RNOs with $k=1,2,3, \ldots$ can be arranged as

$$
\langle x \mid \tilde{k}(t)\rangle= \begin{cases}|\uparrow\rangle \tilde{\phi}_{k^{\prime} / 2}(x ; t) & \text { if } k \text { odd } \\ |\downarrow\rangle \underline{\tilde{\phi}}_{k / 2}(x ; t) & \text { if } k \text { even }\end{cases}
$$

so that any consecutive $k$-odd and $k+1$-even RNOs share the same spatial component $\underline{\phi}_{k^{\prime} / 2}(x ; t)$.

\section{Observables}

We are interested in the double-ionization probability of the model $\mathrm{He}$ atom as a function of the laser intensity and in correlated photoelectron spectra, i.e., the probability to find one electron being emitted with momentum $p_{1}$ and the other with $p_{2}$, for laser intensities where NSDI occurs. Both should in principle be calculated via the projection of the wavefunction after the laser pulse on two-electron continuum states of asymptotic momenta $p_{1}$ and $p_{2}$. However, this approach is numerically unfeasible. We will shortly explain how the yields and spectra are calculated in a less rigorous but sufficiently accurate manner in this work.

\section{Ionization probabilities}

An efficient way to calculate ionization probabilities from the two-electron wavefunction $\Phi\left(x_{1} x_{2}\right)$ after the laser pulse is based on the integration of the probability density $\left|\Phi\left(x_{1} x_{2}\right)\right|^{2}$ over certain spatial regions,

$$
\begin{aligned}
P^{0} & =\iint_{\left|x_{1}\right|,\left|x_{2}\right|<a} \mathrm{~d} x_{1} \mathrm{~d} x_{2}\left|\Phi\left(x_{1} x_{2}\right)\right|^{2}, \\
P^{2+} & =\iint_{\left|x_{1}\right|,\left|x_{2}\right| \geq a} \mathrm{~d} x_{1} \mathrm{~d} x_{2}\left|\Phi\left(x_{1} x_{2}\right)\right|^{2}, \\
P^{1+} & =1-P^{0}-P^{2+}
\end{aligned}
$$

where we made use of the fact that $P^{0}+P^{1+}+P^{2+}=1$. The parameter $a>0$ should be sufficiently large such that the probabilities $P^{1+}$ and $P^{2+}$ are negligible for the groundstate and singly-excited eigenstates. On the other hand, $a$ should not be too large so that the probability density describing ionization does not need too much time to leave the neutral-He region $\left|x_{1}\right|,\left|x_{2}\right|<a$. For our model we chose $a=6$.

As for a two-electron system $\left|\Phi\left(x_{1} x_{2}\right)\right|^{2}=$ $\gamma_{2}\left(x_{1} x_{2}, x_{1} x_{2}\right)$, Eqs. (24) and (25) are read in terms of RNOs:

$$
\begin{aligned}
P^{0}= & \sum_{i j k l} \tilde{\underline{x}}_{2, i j k l} \int_{-a}^{a} \mathrm{~d} x_{1} \tilde{\phi}_{i}\left(x_{1}\right) \underline{\tilde{\phi}}_{k}^{*}\left(x_{1}\right) \\
& \times \int_{-a}^{a} \mathrm{~d} x_{2} \tilde{\phi}_{j}\left(x_{2}\right) \underline{\tilde{\phi}}_{l}^{*}\left(x_{2}\right), \\
P^{2+}= & \sum_{i j k l} \tilde{\tilde{x}}_{2, i j k l} \int_{\left|x_{1}\right| \geq a} \mathrm{~d} x_{1} \underline{\tilde{\phi}}_{i}\left(x_{1}\right) \tilde{\tilde{\phi}}_{k}^{*}\left(x_{1}\right) \\
& \times \int_{\left|x_{2}\right| \geq a} \mathrm{~d} x_{2} \underline{\tilde{\phi}}_{j}\left(x_{2}\right) \underline{\tilde{\phi}}_{l}^{*}\left(x_{2}\right) .
\end{aligned}
$$

Note that in the two-electron case this form is equivalent to first reconstructing the wavefunction (which is possible for two electrons [25]) and then using Eqs. (24) and (25).

\section{Momentum distributions}

A numerically efficient method to calculate correlated double-ionization photoelectron spectra is to multiply the twoelectron wavefunction by a mask function $f\left(x_{1} x_{2}\right)$, which removes the parts representing $\mathrm{He}^{+}$and neutral $\mathrm{He}$ :

$$
\Phi^{2+}\left(x_{1} x_{2}\right) \simeq f\left(x_{1} x_{2}\right) \Phi\left(x_{1} x_{2}\right) .
$$

Here, we chose $f\left(x_{1} x_{2}\right)=f\left(x_{1}\right) f\left(x_{2}\right)$, with $f(x)=$ $1 / \sqrt{1+\mathrm{e}^{-c(|x|-a)}}$ and $c=1.25$ [29]. After Fourier transforming $\Phi^{2+}\left(x_{1} x_{2}\right)$ to momentum space,

$$
\Phi^{2+}\left(p_{1} p_{2}\right)=\frac{1}{2 \pi} \int \mathrm{d} x_{1} \int \mathrm{d} x_{2} \Phi^{2+}\left(x_{1} x_{2}\right) e^{-\mathrm{i}\left(p_{1} x_{1}+p_{2} x_{2}\right)},
$$

the double-ionization photoelectron spectrum is obtained as

$$
\rho^{2+}\left(p_{1} p_{2}\right)=2\left|\Phi^{2+}\left(p_{1} p_{2}\right)\right|^{2} .
$$


In our TDRNOT treatment we proceed analogously by first defining

$$
\gamma_{2}^{2+}\left(x_{1} x_{2}, x_{1}^{\prime} x_{2}^{\prime}\right)=f\left(x_{1} x_{2}\right) f^{*}\left(x_{1}^{\prime} x_{2}^{\prime}\right) \gamma_{2}\left(x_{1} x_{2}, x_{1}^{\prime} x_{2}^{\prime}\right),
$$

whose Fourier transform is $\gamma_{2}^{2+}\left(p_{1} p_{2}, p_{1}^{\prime} p_{2}^{\prime}\right)$. Then,

$$
\rho^{2+}\left(p_{1} p_{2}\right)=2 \gamma_{2}^{2+}\left(p_{1} p_{2}, p_{1} p_{2}\right),
$$

which can be written as

$$
\begin{aligned}
& \rho^{2+}\left(p_{1} p_{2}\right) \\
& \simeq 2 \sum_{i j k l} \tilde{\tilde{x}}_{2, i j k l} \tilde{\tilde{\phi}}_{i}^{+}\left(p_{1}\right) \underline{\tilde{\phi}}_{j}^{+}\left(p_{2}\right)\left\{\underline{\tilde{\phi}}_{k}^{+}\left(p_{1}\right) \underline{\tilde{\phi}}_{l}^{+}\left(p_{2}\right)\right\}^{*}
\end{aligned}
$$

where

$$
\underline{\tilde{\phi}}_{i}^{+}\left(p_{j}\right)=\frac{1}{\sqrt{2 \pi}} \int \mathrm{d} x_{j} f(x) \underline{\tilde{\phi}}_{i}\left(x_{j}\right) e^{-\mathrm{i} x_{j} p_{j}} .
$$

We thus have an explicit construction for $\rho^{2+}\left(p_{1} p_{2}\right)$ in terms of RNOs. Note that in TDDFT such a construction in terms of Kohn-Sham orbitals is unknown [29].

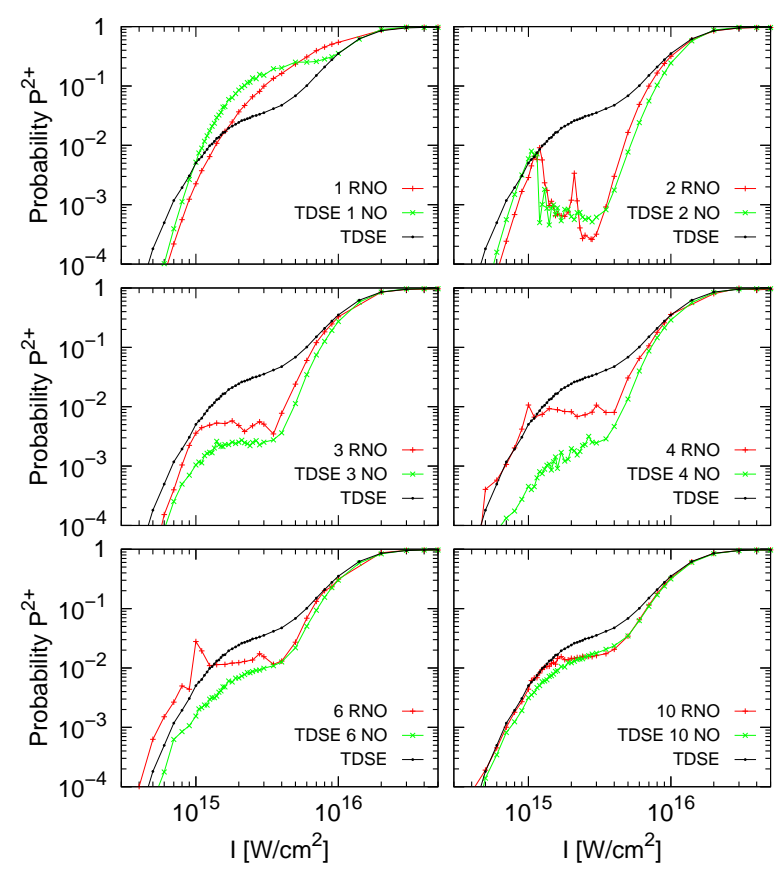

FIG. 1. (Color online) Double-ionization probability vs laser intensity. TDRNOT results with $N=1,2,3,4,6,10$ spatial RNOs (red, +) are compared with the exact TDSE result (black, dots) and with the ionization probability reconstructed using the first $N$ exact NOs calculated from the exact TDSE wavefunction (green, $\times$ ).

\section{RESULTS AND DISCUSSION}

We consider an 800-nm $(\omega=0.058)$ linearly polarized $N_{\text {cyc }}=3$-cycle $\sin ^{2}$-shaped laser pulse of duration $T=$
$2 \pi N_{\text {cyc }} / \omega$. The vector potential in dipole approximation reads

$$
A(t)=\hat{A} \sin ^{2}\left(\frac{\omega t}{2 N_{\text {cyc }}}\right) \sin (\omega t) \quad \text { for } 0 \leq t \leq T
$$

and zero otherwise. The numerical grids for both the TDSEbenchmark and TDRNOT calculations covered \pm 1500 a.u. in the spatial directions.

\section{A. Ionization yields}

Figure 1 shows the double-ionization probability $P^{2+}$ as a function of the laser intensity for different numbers of spatial RNOs $N$ between 1 (upper-left panel) and 10 (lower-right panel). For comparison, the exact TDSE result is included in black in all panels. The nonmonotonic behavior of the first derivative of this exact $P^{2+}$ curve in the region around $2 \times 10^{15} \mathrm{~W} / \mathrm{cm}^{2}$ gives rise to the celebrated NSDI knee.

A TDRNOT calculation with $N=1$ RNO per spin yields a featureless $P^{2+}$ curve, as seen in the upper-left panel of Fig. 1. In fact, in the case of a two-electron spin-singlet system, a single NO per spin is equivalent to TDHF or TDDFT in exchange-only approximation, for which it is already known that the NSDI knee is not reproduced [9-12].

Truncating the number of RNOs in a TDRNOT calculation introduces an error in the propagation of the RNOs [25]. This error should be distinguished from the error that arises alone due to the fact that only a finite number of NOs is taken into account for the calculation of an observable. We do this by determining all exact NOs from the exact TDSE wavefunction but consider only the $N$ most dominant of them to calculate the observable $P^{2+}$. The respective results are also shown in Fig. 1. For $N=1$ this procedure gives a result very different from the TDRNOT with $N=1$. There is even already a knee in the TDSE-1-NO result, albeit a quantitatively wrong one. Both TDRNOT with a single RNO and the TDSE-1-NO curve show a wrong slope in the limit of low laser intensity.

For $N=2$ NOs per spin (upper-right panel) a knee appears also in the TDRNOT result. It is exaggerated and jaggedly structured, and underestimates the $P^{2+}$ yield. A similar behavior with two orbitals was observed in extended Hartree-Fock treatments $[9,21]$ and with the so-called "crapola" model [22], where an "inner" and an "outer" orbital is postulated.

With increasing $N$ the agreement between TDRNOT results and TDSE improves. For $N=10$ the truncation error in the propagation of the RNOs is small enough to give almost the same probability $P^{2+}$ as if it was calculated with the first $N=10$ exact NOs.

Clearly, our TDRNOT approach is only attractive if $N$ can be kept reasonably small. We have shown in Refs. [24, 25] how, with a few RNOs, doubly excited states, autoionization, and Rabi flopping can be described using TDRNOT. Unfortunately, NSDI is more demanding in $N$, meaning that NSDI is highly correlated, and thus many more NOs than particles are required. Moreover, note that although NSDI is a huge effect 


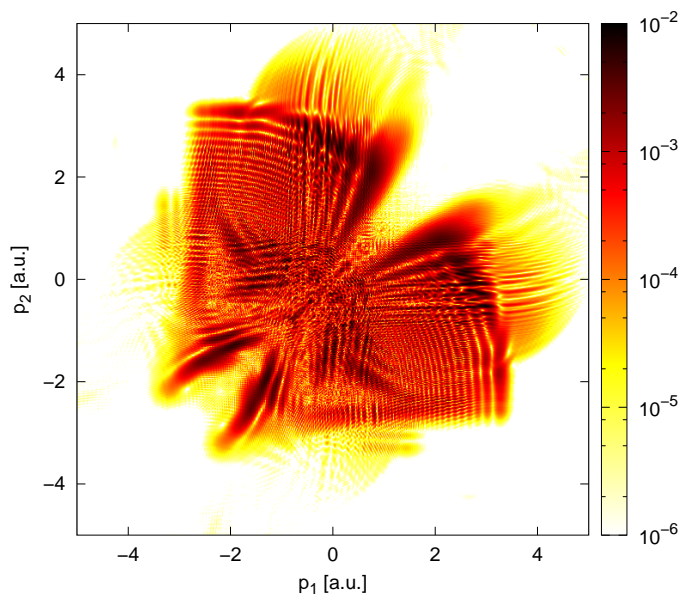

FIG. 2. (Color online) TDSE-benchmark two-electron photoelectron spectrum $\rho^{2+}\left(p_{1} p_{2}\right)$ at $I=2.25 \times 10^{15} \mathrm{~W} / \mathrm{cm}^{2}$.

on the $P^{2+}$ level, it is a small effect compared to the probability for single-ionization $P^{1+}$, and small effects on an absolute scale are captured by NOs with small ONs. The dominant NOs are mainly "responsible" for single ionization, or no ionization at all. In that respect it is remarkable to achieve an agreement such as the one shown for $N=10$ spatial RNOs in Fig. 1. We are not aware of any TDCI or TDMCHF calculation that achieved such an agreement, let alone with only ten basis functions.
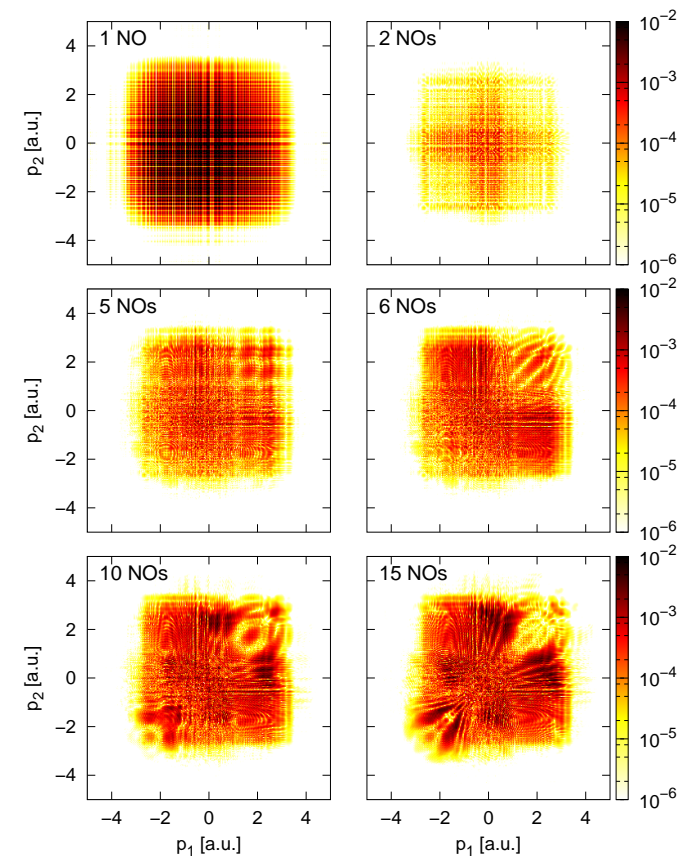

FIG. 3. (Color online) $\rho^{2+}\left(p_{1} p_{2}\right)$ at $I=2.25 \times 10^{15} \mathrm{~W} / \mathrm{cm}^{2}$ obtained from the $N=1,2,5,6,10,15$ dominant, exact spatial NOs calculated from the exact TDSE wavefunction after the laser pulse.

\section{B. Two-electron momentum distribution}

Correlated photoelectron spectra contain more information than ionization probabilities. In general, the "more differential" an observable is, the harder it is to reproduce by some approximate method because the dynamic range to be accurately covered increases. An additional, conceptual challenge arises with TDDFT because $\rho^{2+}\left(p_{1} p_{2}\right)$ is an unknown functional of the single-particle density, and simple approximations fail [29].

Figure 2 shows the TDSE benchmark result for $\rho^{2+}\left(p_{1} p_{2}\right)$ at $I=2.25 \times 10^{15} \mathrm{~W} / \mathrm{cm}^{2}$, i.e., in the NSDI intensity regime. The butterfly structure indicating electrons emitted into the same direction is characteristic of NSDI [1,2] and has been essential to identify rescattering as its origin.

From the TDSE benchmark we know that the first thousand exact NOs have ONs $>10^{-15}$. The question is how many NOs are needed to recover the butterfly structure seen in Fig. 2. Figure 3 shows that with the first 15 exact NOs from the TDSE simulation the butterfly structure of Fig. 2 emerges, but details are still not accurately represented over the 4 orders of magnitude dynamic range shown. However, it is sufficient for the purpose of validating TDRNOT with a reasonably small number of RNOs. Up to $N=5$, mainly uncorrelated, gridlike horizontal and vertical structures are visible. From $N=6$ on, however, clear correlated structures appear, first in the first quadrant $p_{1}, p_{2}>0$.
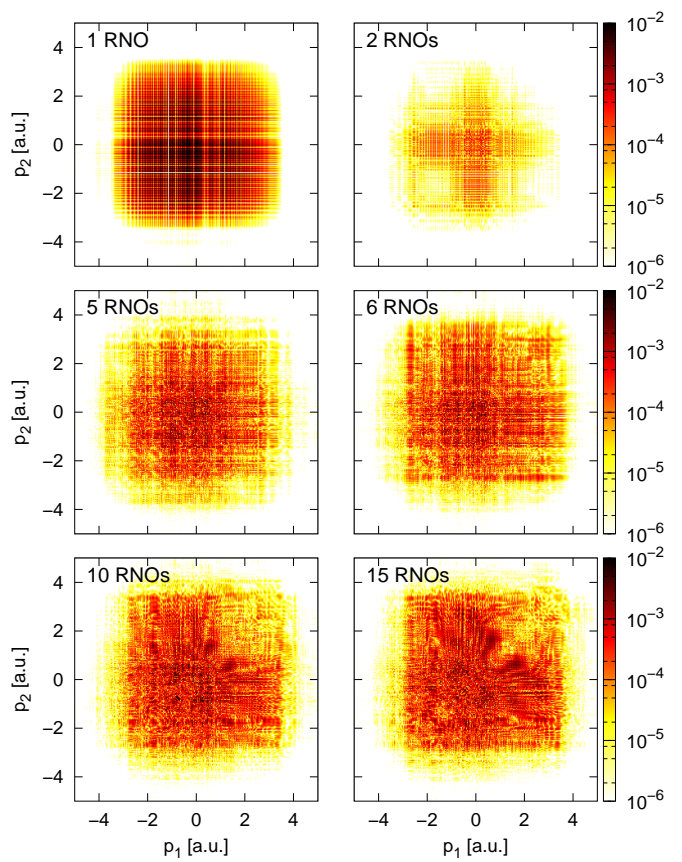

FIG. 4. (Color online) $\rho^{2+}\left(p_{1} p_{2}\right)$ at $I=2.25 \times 10^{15} \mathrm{~W} / \mathrm{cm}^{2}$ obtained from TDRNOT with $N=1,2,5,6,10,15$ spatial RNOs.

Figure 4 shows the corresponding TDRNOT result with $N$ RNOs per spin propagated. Again, the differences between the benchmark results in Fig. 3 and TDRNOT in Fig. 4 are due to the truncation error in the number of propagated RNOs. 
This truncation error severely spoils the correlation structure in the first quadrant; only for $N=15$ does it start to emerge. In order to reproduce, say, the lower-right spectrum in Fig. 3, one would need to propagate about 50 RNOs in TDRNOT. This is prohibitive with our current implementation of solving the nonlinear EOM (12). We found, for instance, that apart from the expected increase of the numerical effort there is the additional complication that the time step needs to be reduced with increasing $N$.

Because of the truncation error, the $N$ th of the (according ON ordered) $N$ dominant spatial RNOs is expected to be most defective. Thus it may make sense to propagate more RNOs than are actually used to calculate observables. Figure 5 shows results where $N=15$ RNOs per spin were propagated but only $N=5$ and 6 were used for the calculation of the photoelectron spectra. One sees that the agreement with the two corresponding middle-row spectra in Fig. 3 is much better than in Fig. 4.
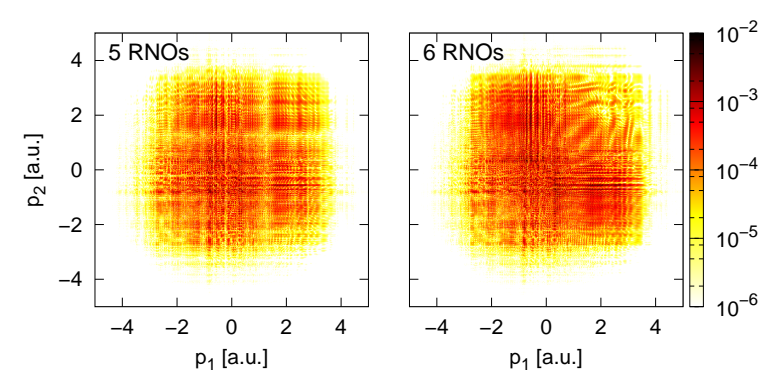

FIG. 5. (Color online) $\rho^{2+}\left(p_{1} p_{2}\right)$ at $I=2.25 \times 10^{15} \mathrm{~W} / \mathrm{cm}^{2}$ obtained from TDRNOT with $N=15$ spatial RNOs propagated but only $N=5$ and 6 used to calculate $\rho^{2+}\left(p_{1} p_{2}\right)$.

\section{Numerical effort}

The computational time $\tau\left(N, N_{x}\right)$ required for a TDRNOT propagation using $N$ NOs on $N_{x}$ spatial grid points scales as

$$
\tau\left(N, N_{x}\right) \sim \alpha N^{2} N_{x} \log N_{x}+\beta N^{3} N_{x}
$$

for a fixed time step. The first term on the right-hand side arises from the calculation of the potentials in (15) using the fast Fourier transform, and the second term from the evaluation of the required matrix elements in (14) [39]. The compu- tational costs of the corresponding operations are taken into account by the constant factors $\alpha$ and $\beta$.

The computational times $\tau(N)=\tau(N, 6000)$ required for one laser intensity using 1 (equivalent to TDHF), 2, and 6 NOs were $\tau(1) \approx 1.3 \mathrm{~min}, \tau(2) \approx 5.6 \mathrm{~min}$, and $\tau(6) \approx 40 \mathrm{~min}$, respectively, on a single core of an i5-3570 processor. This shows that the $N^{2}$ term in (34) is dominating. Compared to the TDSE calculation, where $\tau_{\text {TDSE }}=12 \mathrm{~h}$, TDRNOT thus performs faster by a factor of 550,128 , and 18 , respectively.

Unfortunately, the time step in our present TDRNOT implementation needs to be decreased with increasing $N$ to achieve converged results. For example, for $N=10$ NOs, $\Delta t(10)=0.0016$ was used, whereas in the TDSE simulation $\Delta t=0.075$ was sufficient. This leads to a total computation time of $\tau(10)=30 \mathrm{~h}>\tau_{\mathrm{TDSE}}$ and $\tau(15)=22 \mathrm{~d}$. Hence, improving our TDRNOT scheme to allow for larger time steps is desirable. However, note that for more than two particles the small TDRNOT time step is harmless anyway compared to the exponential scaling of the TDSE wavefunction.

\section{CONCLUSION AND OUTLOOK}

In summary, we reproduced the nonsequential doubleionization knee for a He-model atom starting from the spinsinglet ground state using the recently introduced timedependent renormalized-natural-orbital theory. The equations of motion for the renormalized-natural-orbitals are exact in the two-electron case. This is because the expansion of the time-dependent two-body density matrix in natural orbitals is known exactly. Only the practical limitation in the number of spatial orbitals $N$ forces us to restrict ourselves to $N<20$. Correlated structures in the photoelectron spectra are also reproduced. However, a quantitative agreement with the benchmark spectra obtained from the solution of the corresponding two-electron time-dependent Schrödinger equation can only be achieved with more orbitals.

Current work is devoted to the application of timedependent renormalized-natural-orbital theory to He in full dimensionality, to more electrons, and to the mitigation of the truncation error via better-suited boundary conditions.

\section{ACKNOWLEDGMENT}

This work was supported by the SFB 652 of the German Science Foundation (DFG).
[1] W. Becker, X. Liu, P. J. Ho, and J. H. Eberly, Rev. Mod. Phys. 84, 1011 (2012).

[2] C. F. de Morisson Faria and X. Liu, Journal of Modern Optics 58, 1076 (2011).

[3] T. Weber, M. Weckenbrock, A. Staudte, L. Spielberger, O. Jagutzki, V. Mergel, F. Afaneh, G. Urbasch, M. Vollmer, H. Giessen, and R. Dörner, Phys. Rev. Lett. 84, 443 (2000).
[4] R. Moshammer, B. Feuerstein, W. Schmitt, A. Dorn, C. D. Schröter, J. Ullrich, H. Rottke, C. Trump, M. Wittmann, G. Korn, K. Hoffmann, and W. Sandner, Phys. Rev. Lett. 84, 447 (2000).

[5] T. Weber, H. Giessen, M. Weckenbrock, G. Urbasch, A. Staudte, L. Spielberger, O. Jagutzki, V. Mergel, M. Vollmer, and R. Dörner, Nature 405, 658 (2000). 
[6] B. Feuerstein, R. Moshammer, D. Fischer, A. Dorn, C. D. Schröter, J. Deipenwisch, J. R. Crespo Lopez-Urrutia, C. Höhr, P. Neumayer, J. Ullrich, H. Rottke, C. Trump, M. Wittmann, G. Korn, and W. Sandner, Phys. Rev. Lett. 87, 043003 (2001).

[7] M. Kübel, K. J. Betsch, N. G. Kling, A. S. Alnaser, J. Schmidt, U. Kleineberg, Y. Deng, I. Ben-Itzhak, G. G. Paulus, T. Pfeifer, J. Ullrich, R. Moshammer, M. F. Kling, and B. Bergues, New Journal of Physics 16, 033008 (2014).

[8] A. Rudenko, K. Zrost, B. Feuerstein, V. L. B. de Jesus, C. D. Schröter, R. Moshammer, and J. Ullrich, Phys. Rev. Lett. 93, 253001 (2004).

[9] N. E. Dahlen and R. van Leeuwen, Phys. Rev. A 64, 023405 (2001).

[10] M. Lein and S. Kümmel, Phys. Rev. Lett. 94, 143003 (2005).

[11] M. Petersilka and E. K. U. Gross, Laser Phys. 9, 105 (1999).

[12] F. Wilken and D. Bauer, Phys. Rev. Lett. 97, 203001 (2006).

[13] J. S. Parker, B. J. S. Doherty, K. T. Taylor, K. D. Schultz, C. I. Blaga, and L. F. DiMauro, Phys. Rev. Lett. 96, 133001 (2006).

[14] N. Rohringer, A. Gordon, and R. Santra, Phys. Rev. A 74, 043420 (2006).

[15] A. Karamatskou, S. Pabst, Y.-J. Chen, and R. Santra, Phys. Rev. A 89, 033415 (2014).

[16] S. Sukiasyan, C. McDonald, C. Van Vlack, C. Destefani, T. Fennel, M. Ivanov, and T. Brabec, Phys. Rev. A 80, 013412 (2009).

[17] J. Zanghellini, M. Kitzler, T. Brabec, and A. Scrinzi, Journal of Physics B: Atomic, Molecular and Optical Physics 37, 763 (2004).

[18] J. Caillat, J. Zanghellini, M. Kitzler, O. Koch, W. Kreuzer, and A. Scrinzi, Phys. Rev. A 71, 012712 (2005).

[19] O. Koch, W. Kreuzer, and A. Scrinzi, Applied Mathematics and Computation 173, 960 (2006).

[20] D. Hochstuhl, S. Bauch, and M. Bonitz, Journal of Physics: Conference Series 220, 012019 (2010).
[21] N. A. Nguyen and A. D. Bandrauk, Phys. Rev. A 73, 032708 (2006).

[22] J. B. Watson, A. Sanpera, D. G. Lappas, P. L. Knight, and K. Burnett, Phys. Rev. Lett. 78, 1884 (1997).

[23] W. Kohn, Rev. Mod. Phys. 71, 1253 (1999).

[24] M. Brics and D. Bauer, Phys. Rev. A 88, 052514 (2013).

[25] J. Rapp, M. Brics, and D. Bauer, Phys. Rev. A 90, 012518 (2014).

[26] D. Bauer, Phys. Rev. A 56, 3028 (1997).

[27] D. G. Lappas and R. van Leeuwen, J. Phys. B 31, L249 (1998).

[28] M. Lein, E. K. U. Gross, and V. Engel, Phys. Rev. Lett. 85, 4707 (2000).

[29] F. Wilken and D. Bauer, Phys. Rev. A 76, 023409 (2007).

[30] P.-O. Löwdin, Phys. Rev. 97, 1474 (1955).

[31] A. J. Coleman, Rev. Mod. Phys. 35, 668 (1963).

[32] A. Coleman and V. Yukalov, Reduced Density Matrices, Coulson's Challenge, Springer Lecture Notes in Chemistry 72 (Springer, Berlin, 2000).

[33] K. Pernal, O. Gritsenko, and E. J. Baerends, Phys. Rev. A 75, 012506 (2007).

[34] H. Appel, Time-Dependent Quantum Many-Body Systems: Linear Response, Electronic Transport, and Reduced Density Matrices, Ph.D. thesis, Freie Universität Berlin (2007).

[35] K. J. H. Giesbertz, Time-Dependent One-Body Reduced Density Matrix Functional Theory, Ph.D. thesis, Free University Amsterdam (2010).

[36] H. Appel and E. K. U. Gross, EPL (Europhysics Letters) 92, 23001 (2010).

[37] N. Helbig, J. Fuks, I. Tokatly, H. Appel, E. Gross, and A. Rubio, Chemical Physics 391, 1 (2011).

[38] K. J. H. Giesbertz, O. V. Gritsenko, and E. J. Baerends, The Journal of Chemical Physics 136, 094104 (2012).

[39] Note that, thanks to the sparsity of $\gamma_{2, i j k l}$ in (16), not all combinations of $\mathrm{NO}$ indices contribute. 